\title{
Students'Tripartite Efficacy Beliefs in High School Physical Education: Within- and Cross-Domain Relations With Motivational Processes and Leisure-Time Physical Activity
}

\author{
Ben Jackson, ${ }^{1}$ Peter R. Whipp, ${ }^{1}$ K.L. Peter Chua, ${ }^{1}$ \\ James A. Dimmock, ${ }^{1}$ and Martin S. Hagger ${ }^{2}$ \\ ${ }^{1}$ University of Western Australia; ${ }^{2}$ Curtin University
}

\begin{abstract}
Within instructional settings, individuals form relational efficacy appraisals that complement their self-efficacy beliefs. In high school physical education (PE), for instance, students develop a level of confidence in their teacher's capabilities, as well as estimating how confident they think their teacher is in their (i.e., the students') ability. Grounded in existing transcontextual work, we examined the motivational pathways through which students' relational efficacy and self-efficacy beliefs in PE were predictive of their leisure-time physical activity. Singaporean students $(N=990$; age $M=13.95, S D=1.02)$ completed instruments assessing efficacy beliefs, perceptions of teacher relatedness support, and autonomous motivation toward PE, and 2 weeks later they reported their motivation toward, and engagement in, leisure-time physical activity. Structural equation modeling revealed that students reported stronger other-efficacy and RISE beliefs when they felt that their teacher created a highly relatedness-supportive environment. In turn, their relational efficacy beliefs (a) supported their confidence in their own ability, (b) directly and indirectly predicted more autonomous motives for participation in PE, and (c) displayed prospective transcontextual effects in relation to leisure-time variables. By emphasizing the adaptive motivational effects associated with the tripartite constructs, these findings highlight novel pathways linking students' efficacy perceptions with leisure-time outcomes.
\end{abstract}

Keywords: exercise, other-efficacy, relatedness support, relational efficacy, RISE, self-efficacy

Adolescent physical inactivity is a global epidemiological concern (World Health Organization, 2008), not only due to acute effects upon physical and psychosocial health, but also in light of adverse long-term outcomes arising from the carryover of inactivity from adolescence into adulthood (e.g., Pate et al., 2005). As a result, researchers and policy makers have devoted sustained attention to exploring how physical activity may be better understood and promoted with this population (see Gordon-Larsen, McMurray, \& Popkin, 2000). Due to the vast number of adolescents who participate in some form of compulsory physical education (PE) at school, school-based PE is one setting that has been the continued focus of such work. In summary, studies in this area have shown that positive experiences in high school PE may align with enhanced in-class activity levels, as well as greater concurrent and prospective leisure-time exercise engagement (see Stratton, Fairclough, \& Ridgers, 2008).

Ben Jackson, Peter R. Whipp, K.L. Peter Chua, and James A. Dimmock are with the School of Sport Science, Exercise and Health, University of Western Australia, Crawley, WA, Australia. Martin S. Hagger is with the School of Psychology and Speech Pathology, Curtin University, Perth, WA, Australia.
With an emphasis on adolescent health promotion, a series of studies have explored how students' motivation toward PE may engender desirable leisure-time outcomes (for a review, see Ntoumanis, 2012). According to selfdetermination theory (see Ryan \& Deci, 2008), in which these studies are broadly couched, one's motivation in a given context may range along a self-determination continuum, with highly autonomous motives at one extreme (i.e., participating volitionally in an activity for self-determined reasons, such as fun, interest, and enjoyment), and highly controlled regulation at the other (i.e., where behavior is driven solely by external contingencies). Highlighting the potential for motivation to display transference effects across domains, Hagger and colleagues proposed a transcontextual model (see Hagger \& Chatzisarantis, 2012; Hagger, Chatzisarantis, Culverhouse, \& Biddle, 2003), in which they articulated that autonomous motives in an educational context like PE may predict autonomous motivation toward exercise in a leisure-time context. The model also proposes that autonomous motivation toward leisure-time exercise leads to adaptive outcomes such as increased intentions and engagement in exercise outside school (Hagger \& Chatzisarantis, 2009). In the last decade, research with high school students from Britain (e.g., Hagger et 
al., 2003; Standage, Gillison, Ntoumanis, \& Treasure, 2012), mainland Europe (e.g., Hagger, Chatzisarantis, Barkoukis, Wang, \& Baranowski, 2005), North America (e.g., Wallhead, Hagger, \& Smith, 2010), and Asia (e.g., Hagger et al., 2009) has provided support for these transcontextual proposals, by demonstrating that autonomous motivation toward PE is indeed accompanied by greater autonomous motivation regarding one's leisure-time exercise pursuits, as well as enhanced levels of leisuretime physical activity (LTPA).

In addition to exploring the generality of motivational orientations between domains, transcontextual research has examined the instructional behaviors through which PE teachers may foster students' autonomous motivation. This work has also typically been grounded in self-determination theory, in which it is proposed that autonomous forms of motivation are underpinned by the fulfillment of a series of basic psychological needs (see Ryan \& Deci, 2008), comprising competence (i.e., one's desire to feel capable in one's environment), autonomy (i.e., a desire for volition, input, choice, and a sense of agency in one's pursuits), and relatedness (i.e., one's need to feel connected and close to, as well as valued and understood by, significant others). A number of studies have revealed that students' perceptions of needsupportive teacher behaviors are predictive of increased autonomous motivation for PE (e.g., Taylor \& Ntoumanis, 2007; Tessier, Sarrazin, \& Ntoumanis, 2010); however, that is not to say that additional factors may not also promote adaptive motivational responses. For example, perceptions regarding one's teacher's endorsement of a mastery climate have also been shown to account for improvements in more autonomous forms of PE motivation (Standage, Duda, \& Ntoumanis, 2003). Nonetheless, while it is encouraging that researchers have begun to integrate additional predictors within the transcontextual framework (aside from perceived need support), the motivational properties of a number of other relevant variables remain to be explored.

As one possibility, theory and research indicates that students' PE motivation may also be underpinned, at least in part, by their tripartite efficacy perceptions (Lent \& Lopez, 2002). Within instructional (e.g., teacher-student) scenarios, Lent and Lopez (2002) asserted that in addition to their confidence in their own ability (i.e., self-efficacy; Bandura, 1997), students also form two relational efficacy beliefs regarding their teacher. First, they proposed that students develop a degree of confidence in their teacher's capabilities (e.g., "I've really believe in my PE teacher's ability"), which they referred to as other-efficacy. Second, they contended that students also gauge the extent to which their teacher is confident in their (i.e., the student's) ability. This construct, termed relation-inferred selfefficacy (RISE), reflects a metaperception encompassing students' estimations of their teacher's confidence in them (e.g., "I think my PE teacher really believes in my ability in this class"). Lent and Lopez hypothesized that individuals would be more confident in their own ability when they report favorable relational efficacy beliefs, and also posited that each of the constructs in their tripartite model may independently predict motivational outcomes. In support, within PE and instructor-led physical activity classes, it has been shown that individuals do indeed report increased confidence in their own ability when they hold favorable other-efficacy and RISE beliefs about their teacher/instructor (Jackson, Myers, Taylor, \& Beauchamp, 2012; Jackson Whipp, \& Beauchamp, in press), and also that each of these constructs directly or indirectly predicts adaptive motivational consequences (i.e., effort, enjoyment; Jackson, Myers, et al., 2012).

There have been previous calls for the integration of self-efficacy and self-determination concepts in the study of health promotion (e.g., Rothman, Baldwin, \& Hertel, 2004), and the psychological needs framework that exists within self-determination theory provides theoretical support for a link between students' tripartite efficacy beliefs and autonomous motivation. For instance, self-determination theorists contend that satisfying one's need for competence supports desirable motivational orientations (see Ryan \& Deci, 2008), and thus it is possible that a strong sense of confidence in one's own ability (i.e., self-efficacy) may help to promote autonomous motives for participation in PE. Given that autonomous motivation is also facilitated by a feeling of closeness, trust, and connectedness with significant others (i.e., relatedness), it is possible that students may perceive stronger interpersonal connections with their teacher (leading to greater selfdetermined motivation) when they believe that she or he is highly capable, and/or estimate that the teacher is confident in their ability (cf. Jackson, Grove, \& Beauchamp, 2010). In terms of empirical support for these theorized links, self-efficacy has been shown to align with indicators of self-determined motivation in school-based PE (e.g., enjoyment; Barr-Anderson et al., 2008), as well as enhanced autonomous motivation for other health behaviors, including exercise rehabilitation (Sweet et al., 2009). In addition, in a recent instrument development study, Jackson and colleagues (Jackson, Whipp, Chua, Pengelley, \& Beauchamp, 2012) reported positive correlations between high school students' confidence in their own PE ability and their autonomous motivation toward PE. In comparison with the self-efficacy literature, empirical evidence for the motivational effects of Lent and Lopez's (2002) relational efficacy constructs (i.e., other-efficacy and RISE) is somewhat limited. Jackson, Whipp, et al. (2012) did, however, demonstrate that students' otherefficacy and RISE beliefs correlated positively with autonomous motivation toward PE, as well as participation in LTPA. Taken together, these correlational findings provide some evidence that autonomous motivation may act as an indirect mechanism through which students' PE-based efficacy variables may predict their LTPA (cf. Hagger \& Chatzisarantis, 2012). With that in mind, our aim was to employ structural modeling techniques to examine the direct and indirect prospective relationships between students' efficacy beliefs and autonomous motivation toward $\mathrm{PE}$, as well as their autonomous motivation toward, and engagement in, LTPA. 
In addition to exploring the motivational pathways through which students' tripartite efficacy perceptions support transcontextual outcomes, we drew from theory to also test a potential predictor of students' relational efficacy beliefs. To date, researchers have not explored how teachers' instructional behaviors may promote (or stifle) students' relational efficacy judgments, and in this study we examined the role of perceived teacher relatedness support as a predictor of students' other-efficacy and RISE perceptions. Highly relatedness-supportive classroom environments are characterized by warmth, support, attention, openness, and respect from the teacher, and have been shown to encourage autonomous motivational responses on the part of PE students (e.g., Mouratidis, Vansteenkiste, Sideridis, \& Lens, 2011; Tessier et al., 2010). In addition, it has been demonstrated that a high level of perceived relatedness support within interpersonal contexts also promotes adaptive perceptions about the provider of that supportive behavior (e.g., La Guardia, Ryan, Couchman, \& Deci, 2000; Patrick, Knee, Canevello, \& Lonsbary, 2007). In relation to the tripartite model, therefore, it is plausible that relatedness-supportive behavioral cues might influence individuals' other-efficacy and RISE beliefs (see Lent \& Lopez, 2002; Snyder \& Stukas, 1999). For example, empathy, trust, and rapport building have all been shown to be important characteristics of effective teachers (e.g., Martin \& Dowson, 2009), and so it is possible that when students detect these relatedness-supportive instructional behaviors, this may instill a strong sense of confidence in their teacher's ability. Similarly, the understanding, individualized attention, and supportive feedback that are characteristic of relatedness-supportive teachers may be perceived by students as a sign that their teacher is highly confident in their ability (cf. Jackson, Knapp, \& Beauchamp, 2009). To this point, much of the transcontextual literature has focused solely on the motivational implications of autonomy-supportive teacher behaviors (e.g., Barkoukis, Hagger, Lambropoulos, \& Tsorbatzoudis, 2010; Ntoumanis, 2005), and in comparison, the unique role of relatedness support has received relatively limited attention. Thus, alongside the primary aims outlined previously, this additional aspect of our model enabled us to also obtain novel insight into the perceived instructional behaviors that may predict students' relational efficacy perceptions.

Our hypothesized model, incorporating perceived relatedness support and all proposed relationships between efficacy, motivational, and behavioral variables, is displayed in Figure 1. Beginning with our PE-based variables, we specified predictive pathways between students' perceived relatedness support from their teacher, and their levels of other-efficacy, RISE, and autonomous motivation for PE (i.e., Pathways 1-3 in Figure 1). On the basis of theory and research discussed in the previous section, we hypothesized that when students perceived a highly relatedness-supportive PE environment, they would report greater confidence in their teacher's ability, more favorable estimations of their teacher's confidence in their ability, and greater autonomous motivation

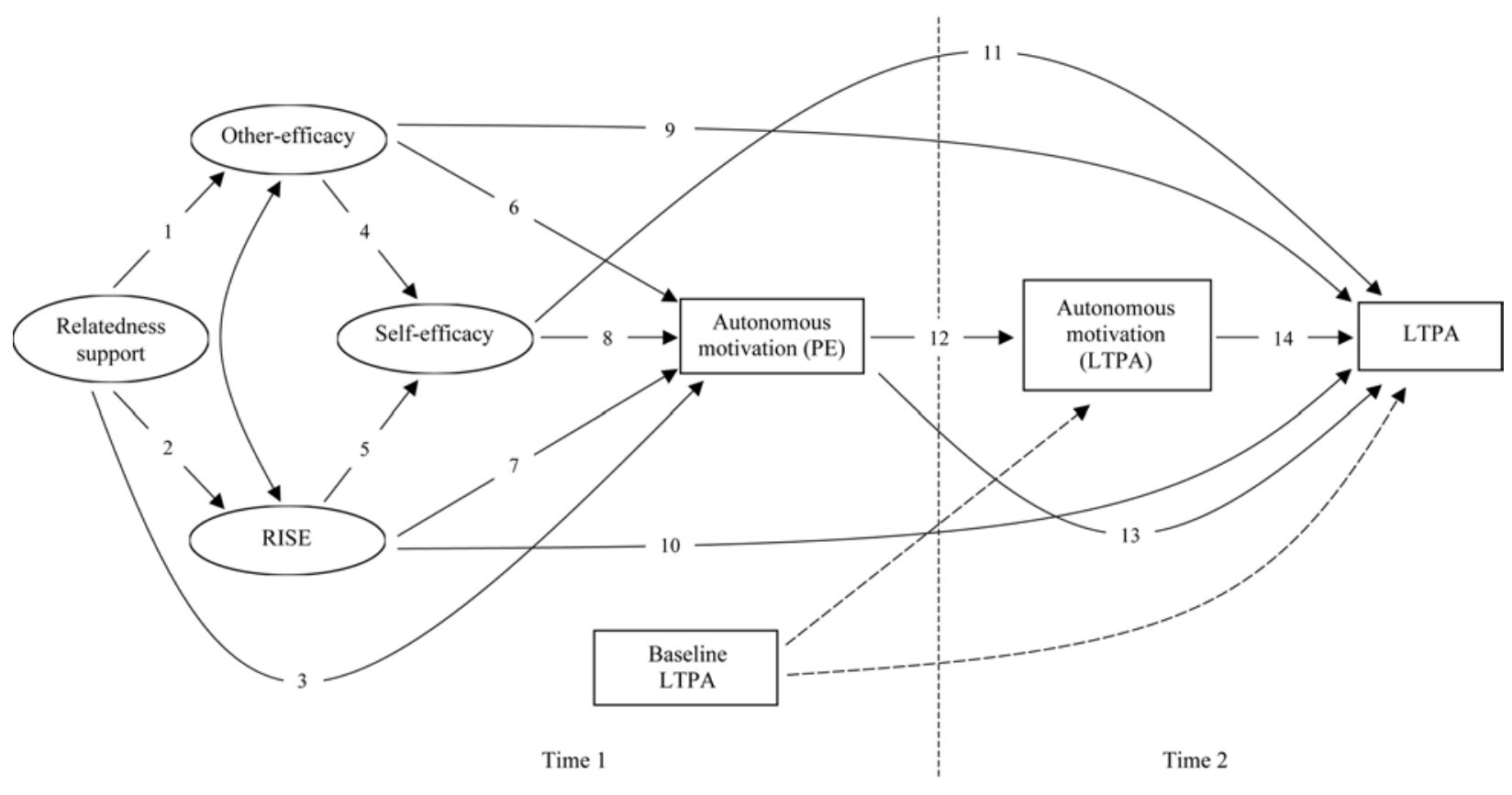

Figure 1 - Hypothesized direct relationships between PE-based (i.e., perceived relatedness support, tripartite efficacy beliefs, autonomous motivation for PE) and leisure-time (i.e., autonomous motivation for LTPA, LTPA) variables. All relationships were hypothesized to be positive in nature. To control for previous activity levels while exploring our hypothesized relationships, we modeled covariance pathways between baseline LTPA and all other Time 1 variables (these covariance pathways are excluded from the figure for clarity), as well as predictive pathways between baseline LTPA and leisure-time (i.e., Time 2) outcomes (see dashed arrows). RISE = relation-inferred self-efficacy. LTPA = leisure-time physical activity. 
toward PE. Guided by Lent and Lopez's (2002) proposals, as well as related tripartite (e.g., Jackson et al., in press) and educational (e.g., Bouchey \& Harter, 2005; Elias \& MacDonald, 2007) research, in Pathways 4 and 5 we forecasted that students' relational efficacy beliefs about their teacher would be positively related to their confidence in their own ability. In light of the potential conceptual links discussed previously, as well as preliminary correlational support for these pathways (Jackson, Whipp, et al., 2012), we also anticipated that students' self-efficacy, other-efficacy, and RISE beliefs would each predict greater autonomous motivation toward PE (i.e., Pathways 6-8).

In terms of transcontextual pathways, we first hypothesized positive effects for students' tripartite perceptions in relation to their LTPA participation (i.e., Pathways 9-11). Conceptually, this allowed us to further examine the correlational associations (Jackson, Whipp, et al., 2012) and direct predictive pathways (Jackson et al., in press) that have been reported previously between PE students' tripartite beliefs and LTPA. In addition, from an analytical perspective, modeling these pathways enabled us to identify the proportion of the total effects between students' tripartite efficacy perceptions and LTPA that were explained by indirect motivational pathways. Second, in light of existing transcontextual work (e.g., Standage et al., 2012), we forecasted that students' autonomous motivation toward PE would generalize across domains and promote greater autonomous motivation toward LTPA (i.e., Pathway 12), as well as increased participation in LTPA (i.e., Pathway 13). Our final pathway, which specified that students' autonomous motivation for LTPA would positively predict engagement in LTPA (i.e., Pathway 14), was derived from previous transcontextual (see Hagger \& Chatzisarantis, 2012) and exercise motivation research (see Edmunds, Ntoumanis, \& Duda, 2007). In summary, although researchers have begun to explore the correlates and predictive effects associated with the tripartite model in educational settings (Jackson, Myers, et al., 2012; Jackson et al., in press), they have yet to (a) examine the instructional behaviors that predict PE students' relational efficacy beliefs (e.g., perceived relatedness support), or (b) draw from the transcontextual model to explore the way in which autonomous motivation may link the tripartite constructs with LTPA.

\section{Method}

\section{Participants}

Complete data were provided by 990 Year 7, 8, and 9 Singaporean students $\left(M_{\text {age }}=13.95, S D=1.02, n_{\text {male }}=528\right.$, $n_{\text {female }}=462$ ) from five state high schools. On average, students participated in $1.50 \mathrm{hr}$ of in-school PE each week $(S D=.32)$, and at baseline, the average LTPA score for this cohort, measured using Godin and Shephard's (1985) instrument (described below), was $39.88(S D=26.45)$. Students were drawn from 30 separate classes (6 per school), resulting in an average class size of 33 students.
All participants declared themselves to be of Singaporean nationality, and the schools selected were representative of an average level of socioeconomic status.

\section{Measures}

Perceived Relatedness Support. Students' perceptions of relatedness support were assessed with the five-item instrument developed for use in high school settings by Standage and colleagues (Standage, Duda, \& Ntoumanis, 2005). Using a scale anchored at 1 (strongly disagree) and 7 (strongly agree), participants responded to statements relating to how they felt about their PE teacher at that moment in time. Items included, "my PE teacher supports me" and "my PE teacher has respect for me." Standage and colleagues (2005) provided evidence for the unidimensionality and predictive utility of the measure derived from this instrument with a high school sample, and we observed an acceptable level of internal consistency for our perceived relatedness support measure $(\alpha=.94)$.

Tripartite Efficacy Beliefs. Students' self-efficacy, other-efficacy, and RISE beliefs were measured using Jackson and colleagues' (Jackson, Whipp, et al., 2012) high school PE tripartite instruments. Using a 1 (no confidence at all) to 5 (complete confidence) response scale, students responded to nine items for each of the tripartite constructs. For self-efficacy, students were instructed to think about their PE class and to "please honestly rate your confidence in your ability at this moment in time to ..." followed by items including, "be physically fit enough to always perform well in PE" and "learn all the skills and activities you are taught, even the most difficult ones." For other-efficacy, students were asked to "please honestly rate your confidence in your PE teacher's ability at this moment in time to ..." and example items included, "be an expert in all the sports and activities you cover in PE" and "provide a variety of activities that makes your PE classes interesting." Finally, students' RISE appraisals were assessed using the exact same nine items that were used to measure self-efficacy. In this instance though, students were instructed to "estimate (or guess) how confident your PE teacher is in your ability in PE at this moment in time." To emphasize the distinction between self-efficacy and RISE perceptions, we also included the statement, "so, we're not focusing on how confident you are; we're focusing on whether you think your PE teacher is confident in you or not.' Jackson, Whipp, et al. (2012) presented support for the internal consistency, factorial validity, and criterion validity of measures derived from these tripartite instruments with a sample of Australian and Singaporean students, as well as evidence of measurement invariance by gender and nationality. In the current study, acceptable alpha coefficients were observed for self-efficacy $(\alpha=.92)$, other-efficacy $(\alpha=.92)$, and RISE $(\alpha=.94)$ measures.

Autonomous Motivation for PE. We used Goudas and colleagues' (Goudas, Biddle, \& Fox, 1994) Perceived 
Locus of Causality (PLOC) questionnaire to measure students' PE-related motivation. Preceded by the stem "I take part in PE classes," participants were instructed to respond honestly to 16 items assessing four distinct motivational regulations, namely, intrinsic motivation (four items, e.g., "because I enjoy learning new skills"), identified regulation (four items, e.g., "because it is important for me to do well in PE"), introjected regulation (four items, e.g., "because I want the teacher to think I'm a good student"), and external regulation (four items, e.g., "because I'll get into trouble if I don't"). In line with Goudas et al.'s (1994) recommendation, a 1 (strongly disagree) to 7 (strongly agree) response format was employed. To calculate students' autonomous motivation toward PE, we used a formula $(2 \times$ intrinsic motivation + identified regulation - introjected regulation $-2 \times$ external regulation) to obtain a single relative autonomy index that weighted individuals' responses across the four distinct motivational regulations. Accordingly, higher scores on this index reflect greater levels of autonomous motivation relative to controlled motivation. Recent investigations have provided support for the reliability and validity of measures derived from the PLOC instrument (e.g., Lonsdale, Sabiston, Taylor, \& Ntoumanis, 2011), as well as evidence for the psychometric properties of measures derived from this instrument specifically with Singaporean students (e.g., Wang, Hagger, \& Liu, 2009).

Autonomous Motivation for LTPA. Students' autonomous motivation for LTPA was assessed using 15 items from Markland and Tobin's (2004) Behavioral Regulation in Exercise Questionnaire-2 (BREQ-2). Using a response scale anchored at 0 (not at all true for me) and 4 (very true for me), students were instructed to consider their reasons for participation in voluntary sport and exercise outside school, and completed items that assessed intrinsic motivation (four items, e.g., "I find exercise a pleasurable activity"), identified regulation (four items, e.g., "I value the benefits of exercise"), introjected regulation (three items, e.g., "I feel guilty when I don't exercise"), and external regulation (four items, e.g., "I feel under pressure from my friends/family to exercise"). Markland and colleagues have previously provided support for the psychometric properties (e.g., factor structure, criterion validity) of measures derived from this instrument (e.g., Markland \& Tobin, 2004), and support for the structural integrity and predictive utility of measures derived from the BREQ-2 with Singaporean students has also been demonstrated (e.g., Jackson, Whipp, et al., 2012). The weighting formula used with the PLOC data was also used with BREQ-2 responses, to create a single score representing students' relative levels of autonomous motivation for LTPA (again, where higher values correspond to greater autonomous motivation relative to controlled motivation).

LTPA. Self-reported LTPA was assessed using the Leisure Time Exercise Questionnaire (LTEQ; Godin \& Shephard, 1985). Definitions and examples for mild, moderate, and vigorous activity categories were provided in line with procedures outlined by Godin and Shephard (1985), and participants were requested to report how many bouts of mild, moderate, and vigorous sport and/or exercise (lasting more than $20 \mathrm{~min}$ ) that they had voluntarily completed in their leisure time over the previous 7 days. Participants were asked to exclude any curriculum-based (e.g., PE) as well as any compulsory school-based physical activity in which they had participated (e.g., physical activity interventions). Using the original formula provided by Godin and Shephard (i.e., $9 \times$ number of vigorous bouts $+5 \times$ number of moderate bouts $+3 \times$ number of mild bouts), a total LTPA score was calculated. The LTEQ has consistently demonstrated adequate psychometric properties and predictive validity with cohorts similar to those used in the present investigation (e.g., Hagger et al., 2003, 2005).

\section{Procedure}

Having obtained ethical approval from the lead author's institution (and after having data collection procedures approved by the Singapore Ministry of Education), letters were distributed to five high school principals describing the nature of the project. Principals were asked to contact the research assistant, at which time they were provided with detailed information about the requirements of the investigation. Principals were notified that student involvement was voluntary, and were also provided with information regarding participants' rights (i.e., confidentiality, right to withdraw). After being given the opportunity to ask any questions, principals were invited to provide consent (in loco parentis) for their students to participate in the investigation. Subsequently, appointments were scheduled for the research assistant to visit each school on two separate occasions (separated by 2 weeks) to complete data collection. Data collection sessions were scheduled during morning registration, recess, or lunch, and, at the start of the first session, students were provided with a written information sheet outlining that their participation was entirely voluntary, their right to withdraw or refuse to answer any question, and that all data would remain confidential at all times. In addition, students were assured that their teacher and classmates would not be made aware of their responses at any time, and that their decision about participating in the investigation would not influence their standing or grade in their class in any way. After being given the opportunity to ask questions, students provided informed consent and completed the first part of the questionnaire package. During the first data collection session, which took place at approximately the midpoint of the school year, students initially completed baseline LTPA (covering activity levels over the previous 7 days), relatedness support, other-efficacy, and RISE measures, and following a short break of approximately $10 \mathrm{~min}$ (in an attempt to provide some separation between RISE and self-efficacy measurement), students also completed 
self-efficacy and PE-related motivation instruments. Two weeks later, the research assistant visited each school a second time to obtain student responses for leisure-time variables.

\section{Data Analysis}

A nonstandard structural equation model incorporating latent and nonlatent variables was specified to analyze the data, using Mplus Version 6.12 (Muthén \& Muthén, 1998-2010). Given that students were nested within classes, we implemented a correction for nonindependence of observations based on student clustering (Asparouhov \& Muthén, 2006). Consistent with Figure 1, we specified a single model that included all measurement parameters, as well as all direct and indirect structural pathways between latent (i.e., relatedness support, selfefficacy, other-efficacy, RISE) and single-item observed (i.e., autonomous motivation scores, LTPA) variables. With the exception of autonomous motivation and LTPA variables, we treated all observed data as ordinal and used weighted least squares mean- and variance-adjusted (WLSMV) estimation. Self-efficacy, other-efficacy, and RISE were each represented by nine indicators, and five indicators were used to represent the latent relatedness support variable. To control for the effects of previous LTPA in our model, we specified baseline LTPA as a single-item observed continuous variable. Specifically, we estimated covariance pathways between baseline LTPA and all other variables that were also measured at Time 1 , as well as a predictive pathway between baseline LTPA and Time 2 variables (i.e., autonomous motivation for LTPA, and LTPA). Missing data (comprising $0.9 \%$ of all cases) were handled under the assumption that they were missing at random, and to account for the combination of ordinal and continuous data during estimation, the variance of each of our continuous observed variables was fixed within an appropriate range (by dividing each of these variables by a constant). In line with existing recommendations (Tabachnick \& Fidell, 2007), and in accordance with the available output provided within Mplus, a range of indices were considered when assessing overall (i.e., combined measurement and structural) model fit, namely the $\chi^{2}$ goodness-of-fit index, comparative fit index (CFI), Tucker-Lewis index (TLI), and root mean square error of approximation (RMSEA). We used established norms to assess overall fit, CFI and TLI $\geq .95$, and RMSEA $\leq$ .08 (Hu \& Bentler, 1999).

\section{Results}

\section{Descriptive Statistics, Correlations, and Fit Indices}

Mean (and $S D$ ) scores for latent and observed variables were 4.37 (1.48), 3.51 (0.72), 3.56 (0.76), and 3.49 (0.73) for perceived relatedness support, self-efficacy, other-efficacy, and RISE, respectively, and 5.71 (6.85),
3.65 (3.63), and 35.73 (23.49) for autonomous motivation for PE, autonomous motivation for LTPA, and LTPA, respectively. Item-level skewness and kurtosis estimates for all latent variable indicators identified no problematic distributional properties. The data were a close overall fit for a single model that simultaneously included all measurement parameters, as well as structural pathways between latent and observed variables, $\chi^{2}(533)=1255.94, p<.001, \mathrm{CFI}=.97, \mathrm{TLI}=.96$, and RMSEA $=.037$ (90\% confidence interval .034 to .040). Standardized factor loadings were in the range .65 to .94 for perceived relatedness support, and .69 to $.84, .71$ to .87 , and .73 to .90 for self-efficacy, other-efficacy, and RISE, respectively.

\section{Main Analyses}

Direct Effects. Analyses revealed a number of significant direct effects between latent and observed variables (see Figure 2). In line with Cohen's (1992) recommended effect size criteria for path coefficients (i.e., $.10=$ small, $.30=$ moderate, $.50=$ large), perceived relatedness support displayed large effects in relation to both relational efficacy beliefs. That is, students reported greater confidence in their PE teacher's ability (i.e., other-efficacy), and more favorable estimations of their teacher's confidence in them (i.e., RISE), when they felt that their teacher provided a high level of relatedness support. In turn, students' other-efficacy and RISE perceptions both positively predicted their confidence in their own ability in PE, displaying a small-to-moderate and a large effect, respectively. Perceived relatedness support, other-efficacy, and self-efficacy (but not RISE) also displayed significant direct effects in relation to students' autonomous motivation for PE. That is, students reported relatively greater self-determined motives for PE (e.g., fun, enjoyment, value) when they felt that their teacher created a highly relatedness-supportive environment, and when they were highly confident in their own or their teacher's capabilities. The effect for perceived relatedness support was small in magnitude; however, we observed a small-to-moderate and a large effect for other-efficacy and self-efficacy, respectively, in relation to autonomous motivation for PE. In terms of transcontextual relationships, small positive direct effects emerged for other-efficacy and RISE (but not selfefficacy) in relation to participation in LTPA. Specifically, students reported greater LTPA when they felt that they had a highly capable PE teacher, and/or when they gauged that their teacher believed strongly in their ability in PE. We also observed a significant relationship (which was indicative of a large effect) between students' autonomous motivation for PE and their autonomous motivation for LTPA, indicating that those students who derived relatively high levels of interest and enjoyment from their PE lessons tended to endorse the same reasons for participation in LTPA. A small-to-moderate significant relationship was also apparent between autonomous motivation for LTPA and LTPA participation. It is worth 
noting that all these predictive effects upon leisuretime outcomes (i.e., autonomous motivation for, and participation in, LTPA; see Pathways 9, 10, 12, and 14) were observed while controlling for the potential effects of baseline activity levels upon these variables. For clarity, our covariate analyses did reveal that baseline activity levels also predicted autonomous motivation toward LTPA, as well as time two LTPA participation (see dashed pathways in Figure 2).

Indirect Effects. Given the potential for numerous indirect pathways within our model, we restricted our focus only to those indirect effects that were relevant to our primary PE (i.e., autonomous motivation for PE) and leisure-time (i.e., LTPA) outcomes. First, other-efficacy $(\beta=.08, S E=.02, p<.001), \operatorname{RISE}(\beta=.34, S E=.03, p$ $<.001)$, and perceived relatedness support $(\beta=.34, S E$ $=.03, p<.001)$ all displayed significant total indirect effects in relation to students' autonomous motivation for PE. As a proportion of the total effect, indirect estimates demonstrated that indirect pathways accounted for $31.8 \%, 96 \%$, and $77.6 \%$ of the effects for other-efficacy, RISE, and relatedness support, respectively, in relation to autonomous motivation in PE. The most notable specific indirect pathways that emerged in these analyses revealed that other-efficacy (i.e., other-efficacy $\rightarrow$ self-efficacy $\rightarrow$ PE motivation: $\beta=.08, S E=.02, p<.001$ ) and RISE (i.e.,
RISE $\rightarrow$ self-efficacy $\rightarrow$ PE motivation: $\beta=.34, S E=$ $.03, p<.001)$ predicted enhanced autonomous motivation for PE via students' confidence in their own ability, and that perceived relatedness support was connected to autonomous motivation for PE through two distinct predictive chains that extended the indirect effects above (i.e., relatedness support $\rightarrow$ other-efficacy $\rightarrow$ self-efficacy $\rightarrow$ PE motivation: $\beta=.05, S E=.01, p<.001$; relatedness support $\rightarrow$ RISE $\rightarrow$ self-efficacy $\rightarrow$ PE motivation: $\beta=$ $.18, S E=.02, p<.001)$.

Several transcontextual indirect effects were also apparent with respect to LTPA (see Table 1 for all possible indirect pathways). Each of the tripartite constructs displayed significant total indirect effects in relation to LTPA, and as a proportion of the total effect, indirect estimates for the tripartite constructs demonstrated that indirect pathways accounted for $74.3 \%, 42.9 \%$, and $40.7 \%$ of the effects for self-efficacy, other-efficacy, and RISE, respectively, in relation to LTPA. The most intricate specific indirect effects for LTPA revealed the existence of two predictive pathways, both of which incorporated students' relatedness support perceptions, relational and self-efficacy beliefs, and both forms of autonomous motivation (i.e., relatedness support $\rightarrow$ other-efficacy/RISE $\rightarrow$ self-efficacy $\rightarrow$ PE motivation $\rightarrow$ LTPA motivation $\rightarrow$ LTPA). As shown in Table 1 , the

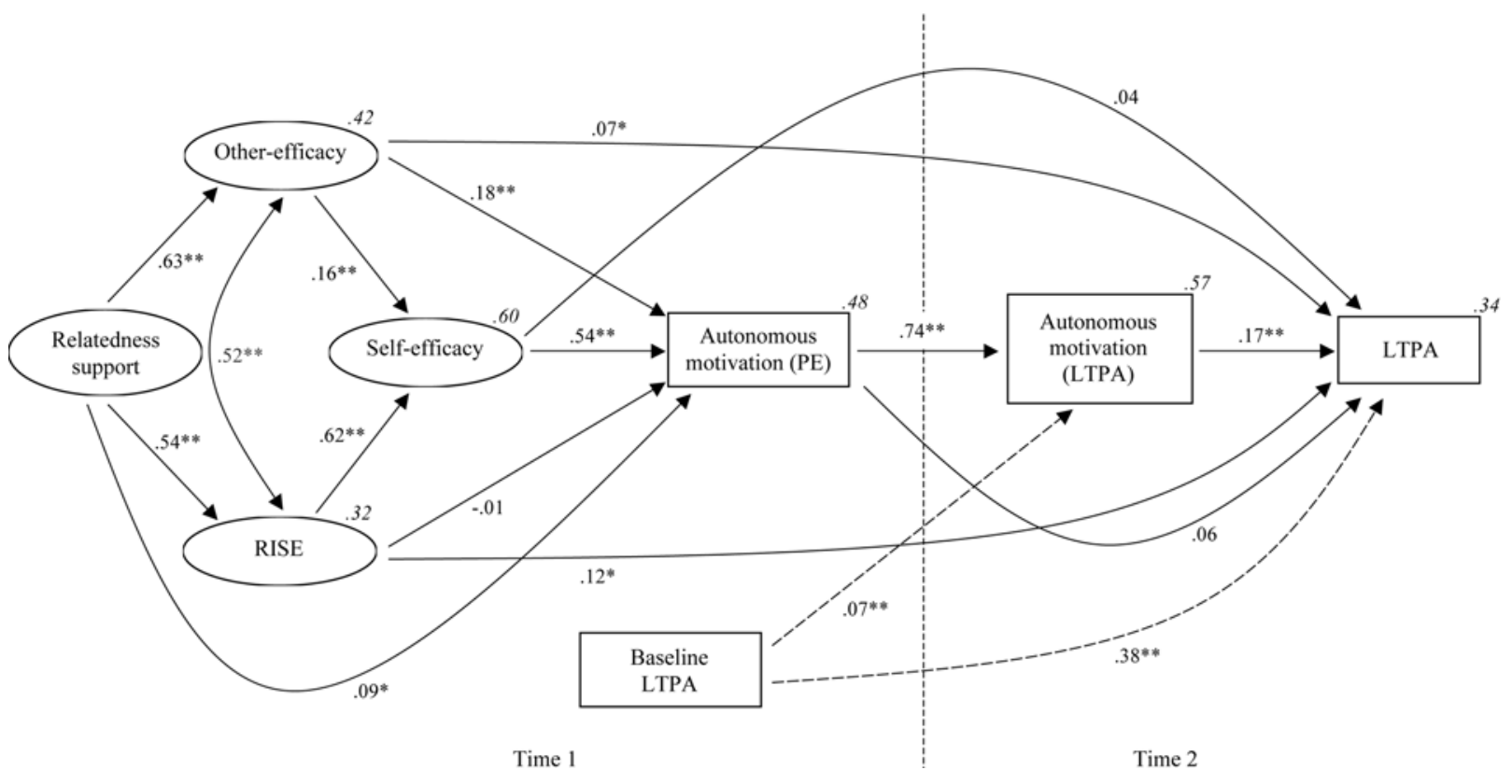

Figure 2 - Direct relationships between PE-based and leisure-time variables. Values above/below arrows represent standardized path coefficients. For interpretational purposes, recommended effect size criteria are $.10=$ small, $.30=$ moderate, and $.50=$ large (Cohen, 1992). All measurement parameters (i.e., categorical indicators) were included alongside structural pathways within this single model, but are excluded from the figure for clarity. Covariance (i.e., double-headed) pathways were estimated between baseline LTPA and all Time 1 variables; however, they were not of primary conceptual interest and so are also excluded from the figure for clarity. The double-headed pathway between other-efficacy and RISE represents the covariance between the residuals of these latent variables. Squared multiple correlations are presented in italics above endogenous variables (all $p<.001$ ), although note that, in the case of Time 2 variables, these values also account for the contribution of baseline LTPA levels (see dashed pathways). RISE $=$ relation-inferred self-efficacy. LTPA $=$ leisure-time physical activity. $* * p<.001 ; * p<.05$. 
Table 1 Standardized Total and Specific Indirect Effects in Relation to Leisure-Time Physical Activity

\begin{tabular}{|c|c|c|c|}
\hline Effect & Estimate & $S E$ & $p$ \\
\hline \multicolumn{4}{|l|}{ Total Indirect Effects } \\
\hline $\mathrm{RS} \rightarrow \mathrm{LTPA}$ & .21 & .02 & $<.001$ \\
\hline $\mathrm{O}-\mathrm{E} \rightarrow \mathrm{LTPA}$ & .06 & .02 & $<.01$ \\
\hline RISE $\rightarrow$ LTPA & .08 & .03 & $<.01$ \\
\hline $\mathrm{S}-\mathrm{E} \rightarrow \mathrm{LTPA}$ & .10 & .02 & $<.001$ \\
\hline PE motivation $\rightarrow$ LTPA & .13 & .04 & $<.01$ \\
\hline \multicolumn{4}{|l|}{ Specific Indirect Effects } \\
\hline $\mathrm{RS} \rightarrow \mathrm{O}-\mathrm{E} \rightarrow \mathrm{LTPA}$ & .05 & .02 & $<.05$ \\
\hline $\mathrm{RS} \rightarrow \mathrm{O}-\mathrm{E} \rightarrow \mathrm{S}-\mathrm{E} \rightarrow \mathrm{LTPA}$ & .01 & .01 & .53 \\
\hline $\mathrm{RS} \rightarrow \mathrm{O}-\mathrm{E} \rightarrow \mathrm{S}-\mathrm{E} \rightarrow$ PE motivation $\rightarrow$ LTPA & .00 & .00 & .36 \\
\hline $\mathrm{RS} \rightarrow \mathrm{O}$-E $\rightarrow$ S-E $\rightarrow$ PE motivation $\rightarrow$ LTPA motivation $\rightarrow$ LTPA & .01 & .00 & $<.01$ \\
\hline $\mathrm{RS} \rightarrow \mathrm{O}-\mathrm{E} \rightarrow$ PE motivation $\rightarrow$ LTPA & .01 & .01 & .36 \\
\hline $\mathrm{RS} \rightarrow \mathrm{O}-\mathrm{E} \rightarrow \mathrm{PE}$ motivation $\rightarrow$ LTPA motivation $\rightarrow$ LTPA & .02 & .01 & $<.05$ \\
\hline $\mathrm{RS} \rightarrow \mathrm{RISE} \rightarrow \mathrm{LTPA}$ & .06 & .03 & $<.05$ \\
\hline $\mathrm{RS} \rightarrow \mathrm{RISE} \rightarrow \mathrm{S}$-E $\rightarrow$ LTPA & .01 & .02 & .54 \\
\hline $\mathrm{RS} \rightarrow \mathrm{RISE} \rightarrow \mathrm{S}-\mathrm{E} \rightarrow$ PE motivation $\rightarrow$ LTPA & .01 & .01 & .33 \\
\hline RS $\rightarrow$ RISE $\rightarrow$ S-E $\rightarrow$ PE motivation $\rightarrow$ LTPA motivation $\rightarrow$ LTPA & .02 & .01 & $<.01$ \\
\hline $\mathrm{RS} \rightarrow \mathrm{RISE} \rightarrow$ PE motivation $\rightarrow$ LTPA & .00 & .00 & .67 \\
\hline RS $\rightarrow$ RISE $\rightarrow$ PE motivation $\rightarrow$ LTPA motivation $\rightarrow$ LTPA & .00 & .00 & .68 \\
\hline $\mathrm{RS} \rightarrow \mathrm{PE}$ motivation $\rightarrow$ LTPA & .01 & .01 & .35 \\
\hline $\mathrm{RS} \rightarrow$ PE motivation $\rightarrow$ LTPA motivation $\rightarrow$ LTPA & .01 & .01 & $<.05$ \\
\hline $\mathrm{O}-\mathrm{E} \rightarrow \mathrm{S}-\mathrm{E} \rightarrow \mathrm{LTPA}$ & .01 & .01 & .53 \\
\hline O-E $\rightarrow$ S-E $\rightarrow$ PE motivation $\rightarrow$ LTPA & .01 & .01 & .36 \\
\hline O-E $\rightarrow$ S-E $\rightarrow$ PE motivation $\rightarrow$ LTPA motivation $\rightarrow$ LTPA & .01 & .00 & $<.01$ \\
\hline O-E $\rightarrow$ PE motivation $\rightarrow$ LTPA & .01 & .01 & .37 \\
\hline O-E $\rightarrow$ PE motivation $\rightarrow$ LTPA motivation $\rightarrow$ LTPA & .02 & .01 & $<.05$ \\
\hline RISE $\rightarrow$ S-E $\rightarrow$ LTPA & .02 & .04 & .53 \\
\hline RISE $\rightarrow$ S-E $\rightarrow$ PE motivation $\rightarrow$ LTPA & .02 & .02 & .33 \\
\hline RISE $\rightarrow$ S-E $\rightarrow$ PE motivation $\rightarrow$ LTPA motivation $\rightarrow$ LTPA & .04 & .01 & $<.01$ \\
\hline RISE $\rightarrow$ PE motivation $\rightarrow$ LTPA & .00 & .00 & .67 \\
\hline RISE $\rightarrow$ PE motivation $\rightarrow$ LTPA motivation $\rightarrow$ LTPA & .00 & .00 & .68 \\
\hline $\mathrm{S}-\mathrm{E} \rightarrow \mathrm{PE}$ motivation $\rightarrow$ LTPA & .03 & .03 & .34 \\
\hline S-E $\rightarrow$ PE motivation $\rightarrow$ LTPA motivation $\rightarrow$ LTPA & .07 & .02 & $<.01$ \\
\hline PE motivation $\rightarrow$ LTPA motivation $\rightarrow$ LTPA & .13 & .04 & $<.01$ \\
\hline
\end{tabular}

Note RS = perceived relatedness support; S-E = self-efficacy; O-E = other-efficacy; RISE = relation-inferred self-efficacy; PE and LTPA motivation $=$ autonomous motivation for PE and LTPA; LTPA = leisure-time physical activity.

various subcomponents (i.e., smaller chains) comprised within these indirect pathways were also significant (e.g., other-efficacy/RISE $\rightarrow$ self-efficacy $\rightarrow$ PE motivation $\rightarrow$ LTPA motivation $\rightarrow$ LTPA). In terms of other unique effects, perceived relatedness support also predicted LTPA through a similar pathway to those above, but that excluded students' confidence in their own ability (i.e., relatedness support $\rightarrow$ other-efficacy $\rightarrow$ PE motivation $\rightarrow$ LTPA motivation $\rightarrow$ LTPA). Finally, favorable relatedness support perceptions were also associated with increased LTPA via less complex pathways, which included only students' other-efficacy or RISE perceptions as intermediary variables (i.e., relatedness support $\rightarrow$ other-efficacy/ RISE $\rightarrow$ LTPA). 


\section{Discussion}

With an emphasis on PE as a context for health promotion, we integrated tripartite efficacy (Lent \& Lopez, 2002) and transcontextual (Hagger \& Chatzisarantis, 2012) proposals to explore the motivational pathways linking students' efficacy beliefs in PE with their LTPA. Analyses revealed that students' relational efficacy appraisals were bolstered by their perceptions about their teacher's relatedness-supportive behaviors, and that the tripartite constructs displayed direct (i.e., other-efficacy, self-efficacy) and/or indirect (i.e., other-efficacy, RISE) effects with respect to autonomous motivation for PE. In addition, direct transcontextual effects were found for other-efficacy and RISE in relation to physical activity, and students' autonomous motivational orientations were responsible for supporting a series of indirect effects between the tripartite constructs and LTPA.

Lent and Lopez (2002) asserted that individuals' relational efficacy cognitions develop out of their general experiences with the target individual, as well as their interpretations of the target's behavior (e.g., his/her competence, feedback, attention, support). As hypothesized, our findings regarding the role of perceived relatedness support demonstrated that students' other-efficacy and RISE perceptions were buoyed when they detected trusting, inclusive, and respectful behaviors from their PE teacher. In line with existing self-determination and transcontextual research (e.g., Ryan, Stiller, \& Lynch, 1994; Tessier et al., 2010), students also endorsed relatively more autonomous (versus controlled) motives for PE when they felt that their teacher created a highly relatedness-supportive environment. From the perspective of integrating tripartite efficacy and transcontextual research, it was noteworthy that relatedness support also indirectly predicted greater autonomous motivation through students' relational (and self-) efficacy perceptions.

In terms of the predictive effects associated with other-efficacy and RISE, our analyses provided insight into how these relational perceptions may support important cognitive, motivational, and behavioral outcomes. Not only did students' relational efficacy beliefs directly predict their confidence in their own ability (cf. Jackson, Myers, et al., 2012), but they also both displayed significant indirect effects in relation to autonomous motivation for PE (via self-efficacy). Alongside these indirect effects, although our hypothesis regarding the direct link between RISE and autonomous motivation was not supported, we did observe that students endorsed relatively more autonomous motives for PE when they were highly confident in their PE teacher's ability. One plausible explanation for the nonsignificant direct effect for RISE is that, for some students, the feeling that one's teacher is highly confident in one's ability might actually induce normative pressures regarding one's involvement in PE. To illustrate this, the perception that "my teacher thinks I'm really capable at PE" might encourage some students to feel that they need to "live up to" that inference during their PE classes. In turn, this perceived pressure might heighten their introjected regulation toward PE (e.g., striving to avoid extrinsically felt outcomes, such as guilt and disapproval from others), while compromising autonomous motives (Ryan \& Deci, 2008). To further examine the motivational effects associated with students' RISE appraisals, researchers are encouraged in future to consider the potential mechanisms that might moderate this relationship. For example, strong RISE beliefs about one's teacher might activate introjected motives only for those students who hold strong chronic orientations toward controlled regulation, as well as those who feel relatively inexperienced, or fear interpersonal rejection, in relation to a given activity.

On the other hand, the within-context motivational effect that was apparent for other-efficacy extends Jackson, Whipp, et al.'s (2012) correlational findings, and is the first time that a direct relationship has been demonstrated between students' relational efficacy beliefs and their autonomous motivation within PE. It has been shown previously that strong other-efficacy perceptions account for more "inclusionary" behaviors and appraisals during one's interactions with a target individual (e.g., responsiveness, attention, enjoyment; Jackson et al., 2009). Thus, these inclusionary orientations might promote improved autonomous motivation through either a generalization process, whereby one's feelings about the teacher translate into more positive feelings about the class, or through an interpersonal process, whereby the teacher detects a student's positive attitude and reciprocates with behaviors (e.g., enthusiasm, support, encouragement) that support student self-determined motivation (see Snyder \& Stukas, 1999).

Aside from the within-context motivational effects that were observed for other-efficacy and RISE, analyses also revealed that individuals' relational efficacy beliefs both directly predicted their participation in LTPA (even when controlling for baseline activity levels). This prospective effect for other-efficacy is consistent with existing tripartite (and proxy) efficacy research, in which it has been shown that exercise engagement is directly predicted by a high level of confidence in one's PE teacher (Jackson et al., in press) or exercise instructor (e.g., Bray, Gyurcsik, Culos-Reed, Dawson, \& Martin, 2001). Indeed, Jackson et al. (in press) contended that a high level of confidence in one's teacher might activate more positive attitudinal responses regarding PE activities, which may account for greater extracurricular participation in those activities (cf. Hilland, Ridgers, Stratton, \& Fairclough, 2011). As for RISE, Lent and Lopez (2002) postulated that when individuals hold favorable metaperceptions in relation to a credible, authority figure (e.g., a teacher), then this may play a role in decision making regarding related opportunities for skill deployment. In other words, individuals might display a tendency to be more approach oriented in a given context in response to strong RISE estimations. Thus, it is possible that when opportunities arise for recreational activity (e.g., a group soccer game or exercise training session), adolescents may be more 
likely to choose to participate when they sense that a credible expert (i.e., their PE teacher) believes strongly in their physical abilities. While these direct transcontextual effects underscore the significance of students' relational efficacy beliefs, the indirect pathways that also linked other-efficacy and RISE with LTPA (via students' selfefficacy and autonomous motivation) provided greater insight into how these variables align with adaptive leisure-time outcomes. In particular, the motivational variables that linked the tripartite constructs with LTPA demonstrated support for transcontextual proposals (e.g., Hagger et al., 2005), and in future researchers might test whether these motivational pathways also facilitate links between students' relational efficacy perceptions and other extracurricular outcomes (e.g., attitudes toward exercise).

Although the literature regarding students' relational efficacy perceptions is at a development stage, wideranging evidence already exists to support the utility of self-efficacy in educational settings (see Zimmerman, 2000). As hypothesized, we observed a direct withincontext effect between students' confidence in their own ability and their level of autonomous motivation. That is, individuals tended to be driven by relatively more self-determined (e.g., interest, fun) rather than extrinsic (e.g., pressure, guilt) motives in PE when they believed strongly in their own capabilities. Self-efficacy in PE was also indirectly connected to LTPA via a prospective pathway that supported the tenets of the transcontextual model (i.e., self-efficacy $\rightarrow$ PE motivation $\rightarrow$ LTPA motivation $\rightarrow$ LTPA). These pathways not only endorse the integration of self-efficacy and self-determination theory concepts, but also align with the literature linking competence perceptions and motivational outcomes in educational pursuits (e.g., Standage et al., 2003).

Nonetheless, it is important to note that neither self-efficacy nor autonomous motivation for PE aligned directly with LTPA. Given the strength of the effects that both these constructs displayed in relation to their most proximal outcomes (see Pathways 8 and 12), it may be somewhat unsurprising that they failed to also exert direct effects in relation to downstream consequences. In the case of autonomous motivation for $\mathrm{PE}$, for instance, it appeared that any resultant effect upon LTPA was due almost exclusively to an indirect pathway that flowed through leisure-time motivation. With regard to self-efficacy, the nonsignificant direct effect (i.e., Pathway 11) that we observed with this Singaporean cohort was inconsistent with the positive relationship that Jackson et al. (in press) recently reported between PE self-efficacy and leisure-time exercise engagement among UK adolescents (see also Shen, McCaughtry, \& Martin, 2008). That said, from a conceptual perspective, the lack of direct effect for self-efficacy may be partly attributable to the significant effect that was apparent between RISE and LTPA. This direct effect for RISE was not evident within Jackson et al.'s UK cohort, and it is possible that, in comparison with students from the UK, Singaporean adolescents'
LTPA may rely to a greater extent on how they feel their teacher appraises their ability, rather than their own view of their capabilities in PE. Lent and Lopez (2002) proposed that RISE beliefs are at their most salient when the target individual represents a highly knowledgeable and credible information source. Relative to UK adolescents, Singaporeans display a stronger cultural predisposition toward respecting, and deferring to, those in positions of authority, such as teachers (see the concept of "power distance"; Hofstede, 2001). As such, it is plausible that this cultural orientation might augment the predictive effects stemming from students' inferences about their teacher's belief in their ability (i.e., RISE), while reducing the predictive import associated with students' confidence in their own ability (cf. Taylor \& Lonsdale, 2010). Future research that accounts for potential moderators of these relationships (e.g., power distance orientations) will enable researchers to address important questions about when and why students' self-efficacy and/or RISE perceptions display (and do not display) direct transcontextual effects upon LTPA.

In terms of design limitations, it is important to note that we obtained self-report measures of physical activity engagement, and it would be valuable in future to incorporate more objective activity measures over extended periods of time (e.g., via the use of accelerometers). It would also be conceptually and practically worthwhile to explore the processes through which students' tripartite efficacy beliefs may generalize across contexts (Bandura, 1997). For instance, it would be fascinating to determine whether (and how) relational perceptions about one's PE teacher might transfer to extracurricular social agents. It is possible, for example, that when students estimate that their teacher is highly confident in their ability in PE, they might also infer that their parents believe strongly in their physical capabilities. Future tripartite efficacy research could also provide novel information regarding the mechanisms associated with Pygmalion effects and reflected appraisal processes in PE (e.g., Trouilloud, Sarrazin, Martinek, \& Guillet, 2002). To illustrate, it would be interesting to determine how the effects of teacher expectations upon student outcomes are filtered through students' RISE perceptions. Alternatively, the magnitude of any effects of teacher expectations upon student outcomes might also be moderated by the extent to which students believe in their teacher's capabilities (see Pornpitakpan, 2004).

Although our data do not allow for insight into causal relations between variables, this is the first study to document how teachers' instructional behaviors may promote students' relational efficacy beliefs, and these findings encourage further tripartite research that examines the role of perceived relatedness support in PE. In particular, intervention programs would be worthwhile that target teachers' relatedness support behaviors, and track changes in students' relational efficacy beliefs, motivational profiles, and subsequent activity patterns. In addition, physical activity researchers might also consider whether contextual factors moderate the extent to which 
other-efficacy and RISE are underpinned by relatednesssupportive instruction. Physical education classes are characterized by a high level of student-teacher interaction, and have been shown, in some instances, to evoke social anxiety and peer acceptance concerns (e.g., Cox, Ullrich-French, Madonia, \& Witty, 2011). As a result, it is possible that a highly relatedness-supportive environment, which emphasizes feelings of comfort, understanding, and trust, is particularly crucial for promoting relational efficacy perceptions in this context. It would be intriguing to explore whether these instructional behaviors may be less pertinent in activity settings where interaction is not as frequent, where performers' skill levels are more advanced, and/or where self-evaluative concerns are not as prevalent.

To conclude, this investigation furthers our understanding of tripartite efficacy and transcontextual processes by revealing support for complementary relations between adolescents' efficacy beliefs, motivational orientations, and physical activity levels. Previous PE work has demonstrated that self-efficacy appraisals align with higher levels of motivation; however, the present findings illustrate that the tripartite constructs may be responsible for promoting higher quality (i.e., autonomous) motivational responses. This study also documented how teachers' instructional behaviors might strengthen students' relational efficacy beliefs, as well as identifying novel indirect pathways through which the tripartite constructs promote important activity-related outcomes. In sum, there appears to be significant potential for future research in pedagogical settings that explores the motivational and health-related implications of students' tripartite efficacy perceptions.

\section{Acknowledgment}

This work was partly supported by a grant to the first author from the Australian Research Council (DE120101006).

\section{References}

Asparouhov, T., \& Muthén, B.O. (2006). Multilevel modeling of complex survey data. In Proceedings of the Joint Statistical Meeting, USA, American Statistical Association Section on Survey Research Methods (pp. 2718-2726), Seattle, WA.

Bandura, A. (1997). Self-efficacy: The exercise of control. New York: Freeman and Company.

Barkoukis, V., Hagger, M.S., Lambropoulos, G., \& Tsorbatzoudis, H. (2010). Extending the trans-contextual model in physical education and leisure-time contexts: Examining the role of basic psychological need satisfaction. The British Journal of Educational Psychology, 80, 647-670. PubMed doi:10.1348/000709910X487023

Barr-Anderson, D.J., Neumark-Sztainer, D., Schmitz, K.H., Ward, D.S., Conway, T.L., Pratt, C., . . Pate, R.R. (2008). But I like PE: Factors associated with enjoyment of physical education class in middle school girls. Research Quarterly for Exercise and Sport, 79, 18-27. PubMed doi :10.5641/193250308X13086753542735
Bouchey, H.A., \& Harter, S. (2005). Reflected appraisals, academic self-perceptions, and math / science performance during early adolescence. Journal of Educational Psychology, 97, 673-686. doi:10.1037/0022-0663.97.4.673

Bray, S.R., Gyurcsik, N.C., Culos-Reed, S.N., Dawson, K.A., \& Martin, K.A. (2001). An exploratory investigation of the relationship between proxy efficacy, self-efficacy and exercise attendance. Journal of Health Psychology, 6, 425-434. doi:10.1177/135910530100600405

Cohen, J. (1992). A power primer. Psychological Bulletin, 112, 155-159. PubMed doi:10.1037/0033-2909.112.1.155

Cox, A.E., Ullrich-French, S., Madonia, J., \& Witty, K. (2011). Social physique anxiety in physical education: Social contextual factors and links to motivation and behavior. Psychology of Sport and Exercise, 12, 555-562. doi:10.1016/j. psychsport.2011.05.001

Edmunds, J.K., Ntoumanis, N., \& Duda, J.L. (2007). Perceived autonomy support and psychological need satisfaction in exercise. In M.S. Hagger \& N.L.D. Chatzisarantis (Eds.), Intrinsic motivation and self-determination in exercise and sport (pp. 35-52). Champaign, IL: Human Kinetics.

Elias, S.M., \& MacDonald, S. (2007). Using past performance, proxy efficacy, and academic self-efficacy to predict college performance. Journal of Applied Social Psychology, 37, 2518-2531. doi:10.1111/j.1559-1816.2007.00268.x

Godin, G., \& Shephard, R.J. (1985). A simple method to assess exercise behavior in the community. Canadian Journal of Applied Sport Sciences, 10, 141-146. PubMed

Gordon-Larsen, P., McMurray, R.G., \& Popkin, B.M. (2000). Determinants of adolescent physical activity and inactivity patterns. Pediatrics, 105, 1-8. doi:10.1542/peds.105.6.e83

Goudas, M., Biddle, S.J.H., \& Fox, K.R. (1994). Perceived locus of causality, goal orientations, and perceived competence in school physical education classes. The British Journal of Educational Psychology, 64, 453-463. PubMed doi:10.1111/j.2044-8279.1994.tb01116.x

Hagger, M.S., \& Chatzisarantis, N.L.D. (2009). Integrating the theory of planned behaviour and self-determination theory in health behaviour: A meta-analysis. British Journal of Health Psychology, 14, 275-302. PubMed doi:10.1348/135910708X373959

Hagger, M.S., \& Chatzisarantis, N.L.D. (2012). Transferring motivation from educational to extramural contexts: A review of the trans-contextual model. European Journal of Psychology of Education, 27, 195-212. doi:10.1007/ s10212-011-0082-5

Hagger, M.S., Chatzisarantis, N.L.D., Barkoukis, V., Wang, C.K.J., \& Baranowski, J. (2005). Perceived autonomy support in physical education and leisure-time physical activity: A cross-cultural evaluation of the trans-contextual model. Journal of Educational Psychology, 97, 376-390. doi:10.1037/0022-0663.97.3.376

Hagger, M.S., Chatzisarantis, N.L.D., Culverhouse, T., \& Biddle, S.J.H. (2003). The processes by which perceived autonomy support in physical education promotes leisuretime physical activity intentions and behavior: A transcontextual model. Journal of Educational Psychology, 95, 784-795. doi:10.1037/0022-0663.95.4.784 
Hagger, M.S., Chatzisarantis, N.L.D., Hein, V., Soós, I., Karsai, I., Lintunen, T., \& Leemans, S. (2009). Teacher, peer and parent autonomy support in physical education and leisure-time physical activity: A trans-contextual model of motivation in four nations. Psychology \& Health, 24, 689-711. PubMed doi:10.1080/08870440801956192

Hilland, T.A., Ridgers, N.D., Stratton, G., \& Fairclough, S.J. (2011). Associations between school physical education correlates and adolescent physical activity. Pediatric Exercise Science, 23, 61-71. PubMed

Hofstede, G. (2001). Culture's consequences: Comparing values, behaviors, institutions, and organizations across nations (2nd ed.). Thousand Oaks, CA: Sage.

Hu, L., \& Bentler, P.M. (1999). Cutoff criteria for fit indexes in covariance structure analysis: Conventional criteria versus new alternatives. Structural Equation Modeling, 6, 1-55. doi:10.1080/10705519909540118

Jackson, B., Grove, J.R., \& Beauchamp, M.R. (2010). Relational efficacy beliefs and relationship quality within coach-athlete dyads. Journal of Social and Personal Relationships, 27, 1035-1050. doi:10.1177/0265407510378123

Jackson, B., Knapp, P., \& Beauchamp, M.R. (2009). The coachathlete relationship: A tripartite efficacy perspective. The Sport Psychologist, 23, 203-232.

Jackson, B., Myers, N.D., Taylor, I.M., \& Beauchamp, M.R. (2012). Relational efficacy beliefs in physical activity classes: A test of the tripartite model. Journal of Sport \& Exercise Psychology, 34, 285-304. PubMed

Jackson, B., Whipp, P.R., \& Beauchamp, M.R. (in press).The tripartite efficacy framework in high school physical education: Trans-contextual generality and direct and indirect prospective relations with leisure-time exercise. Sport, Exercise, and Performance Psychology. doi:10.1037/a0030169

Jackson, B., Whipp, P.R., Chua, K.L.P., Pengelley, R., \& Beauchamp, M.R. (2012). Assessment of tripartite efficacy beliefs within school-based physical education: Instrument development and reliability and validity evidence. Psychology of Sport and Exercise, 13, 108-117. doi:10.1016/j. psychsport.2011.10.007

La Guardia, J.G., Ryan, R.M., Couchman, C.E., \& Deci, E.L. (2000). Within-person variation in security of attachment: A self-determination theory perspective on attachment, need fulfillment, and well-being. Journal of Personality and Social Psychology, 79, 367-384. PubMed doi:10.1037/0022-3514.79.3.367

Lent, R.W., \& Lopez, F.G. (2002). Cognitive ties that bind: A tripartite view of efficacy beliefs in growth-promoting relationships. Journal of Social and Clinical Psychology, 21, 256-286. doi:10.1521/jscp.21.3.256.22535

Lonsdale, C., Sabiston, C.M., Taylor, I.M., \& Ntoumanis, N. (2011). Measuring student motivation for physical education: examining the psychometric properties of the perceived locus of causality questionnaire and the situational motivation scale. Psychology of Sport and Exercise, 12, 284-292. doi:10.1016/j.psychsport.2010.11.003

Markland, D., \& Tobin, V. (2004). A modification to the behavioral regulation in exercise questionnaire to include an assessment of amotivation. Journal of Sport \& Exercise Psychology, 26, 191-196.
Martin, A., \& Dowson, M. (2009). Interpersonal relationships, motivation, engagement, and achievement: Yields for theory, current issues, and practice. Review of Educational Research, 79, 327-365. doi:10.3102/0034654308325583

Mouratidis, A., Vansteenkiste, M., Sideridis, G., \& Lens, W. (2011). Vitality and interest-enjoyment as a function of class-to-class variation in need-supportive teaching and pupils' autonomous motivation. Journal of Educational Psychology, 103, 353-366. doi:10.1037/a0022773

Muthén, L.K., \& Muthén, B.O. (1998-2010). Mplus User's Guide (6th ed.). Los Angeles, CA: Muthén \& Muthén.

Ntoumanis, N. (2005). A prospective study of participation in optional school physical education using a self-determination theory framework. Journal of Educational Psychology, 97, 444-453. doi:10.1037/0022-0663.97.3.444

Ntoumanis, N. (2012). A self-determination theory perspective on motivation in sport and physical education: Current trends and possible future directions. In G.C. Roberts \& D.C. Treasure (Eds.), Advances in Motivation in sport and exercise (3rd ed., pp. 91-128). Champaign, IL: Human Kinetics.

Pate, R.R., Ward, D.S., Saunders, R.P., Felton, G., Dishman, R.K., \& Dowda, M. (2005). Promotion of physical activity in high-school girls: a randomized controlled trial. American Journal of Public Health, 95, 1582-1587. PubMed doi:10.2105/AJPH.2004.045807

Patrick, H., Knee, C.R., Canevello, A., \& Lonsbary, C. (2007). The role of need fulfillment in relationship functioning and well-being: A self-determination theory perspective. Journal of Personality and Social Psychology, 92, 434-457. PubMed doi:10.1037/0022-3514.92.3.434

Pornpitakpan, C. (2004). The persuasiveness of source credibility: A critical review of five decades' evidence. Journal of Applied Social Psychology, 34, 243-281. doi:10.1111/j.1559-1816.2004.tb02547.x

Rothman, A.J., Baldwin, A.S., \& Hertel, A.W. (2004). Selfregulation and behavior change: Distengangling behavioral initiation and behavioral maintenance. In R. Baumeister \& K. Vohs (Eds.), Handbook of self-regulation: Research, theory and applications (pp. 130-148). New York: Guilford Press.

Ryan, R.M., \& Deci, E.L. (2008). Self-determination theory and the role of basic psychological needs in personality and the organization of behavior. In O.P. John, R.W. Robbins, \& L.A. Pervin (Eds.), Handbook of personality: Theory and research (pp. 654-678). New York: Guilford Press.

Ryan, R.M., Stiller, J., \& Lynch, J.H. (1994). Representations of relationships to teachers, parents, and friends as predictors of academic motivation and self-esteem. The Journal of Early Adolescence, 14, 226-249. doi:10.1177/027243169401400207

Shen, B., McCaughtry, N., \& Martin, J. (2008). Urban adolescents' exercise intentions and behaviors: An exploratory study of a trans-contextual model. Contemporary Educational Psychology, 33, 841-858. doi:10.1016/j. cedpsych.2007.09.002

Snyder, M., \& Stukas, A.A. (1999). Interpersonal processes: The interplay of cognitive, motivational, and behavioral activities in social interaction. Annual Review of Psychology, 50, 273-303. PubMed doi:10.1146/annurev.psych.50.1.273 
Standage, M., Duda, J.L., \& Ntoumanis, N. (2003). A model of contextual motivation in physical education: Using constructs from self-determination and achievement goal theories to predict physical activity intentions. Journal of Educational Psychology, 95, 97-110. doi:10.1037/00220663.95.1.97

Standage, M., Duda, J.L., \& Ntoumanis, N. (2005). A test of self-determination theory in school physical education. The British Journal of Educational Psychology, 75, 411-433. PubMed doi:10.1348/000709904X22359

Standage, M., Gillison, F., Ntoumanis, N., \& Treasure, D.C. (2012). Predicting students' physical activity and healthrelated well-being: A prospective cross-domain investigation of motivation across school physical education and exercise settings. Journal of Sport \& Exercise Psychology, 34, 37-60. PubMed

Stratton, G., Fairclough, S.J., \& Ridgers, N. (2008). Physical activity levels during the school day. In A.L. Smith \& S.J.H. Biddle (Eds.), Youth physical activity and sedentary behavior: Challenges and solutions (pp. 321-350). Champaign, IL: Human Kinetics.

Sweet, S.N., Fortier, M.S., Guérin, E., Tulloch, H., Sigal, R.J., Kenny, G.P., \& Reid, R.D. (2009). Understanding physical activity in adults with type 2 diabetes after completing an exercise intervention trial: A mediation model of self-efficacy and autonomous motivation. Psychology Health and Medicine, 14, 419-429. PubMed doi:10.1080/13548500903111806

Tabachnick, B.G., \& Fidell, L.S. (2007). Using multivariate statistics (5th ed.). Boston, MA: Pearson/Allyn \& Bacon.

Taylor, I.M., \& Lonsdale, C. (2010). Cultural differences in the relationships among autonomy support, psychological need satisfaction, subjective vitality, and effort in British and Chinese physical education. Journal of Sport \& Exercise Psychology, 32, 655-673. PubMed
Taylor, I.M., \& Ntoumanis, N. (2007). Teacher motivational strategies and student self-determination in physical education. Journal of Educational Psychology, 99, 747-760. doi:10.1037/0022-0663.99.4.747

Tessier, D., Sarrazin, P., \& Ntoumanis, N. (2010). The effect of an intervention to improve newly qualified teachers' interpersonal style, students' motivation and psychological need satisfaction in sport-based physical education. Contemporary Educational Psychology, 35, 242-253. doi:10.1016/j.cedpsych.2010.05.005

Trouilloud, D.O., Sarrazin, P.G., Martinek, T.J., \& Guillet, E. (2002). The influence of teacher expectations on students' achievement in physical education classes: Pygmalion revisited. European Journal of Social Psychology, 32, 591-607. doi:10.1002/ejsp.109

Wallhead, T.L., Hagger, M.S., \& Smith, D. (2010). Sport education and extra-curricular sport participation: An examination using the trans-contextual model of motivation. Research Quarterly for Exercise and Sport, 81, 442-455. PubMed doi:10.5641/027013610X13088600029256

Wang, C.K.J., Hagger, M.S., \& Liu, W.C. (2009). A crosscultural validation of Perceived Locus of Causality scale in physical education context. Research Quarterly for Exercise and Sport, 80, 313-325. PubMed

World Health Organization. (2008). Physical Inactivity: A Global Public Health Problem. Retrieved 9th March 2011, from http://www.who.int/dietphysicalactivity/ factsheet_inactivity/en/index.html

Zimmerman, B.J. (2000). Self-efficacy: An essential motive to learn. Contemporary Educational Psychology, 25, 82-91. PubMed doi:10.1006/ceps.1999.1016

Manuscript submitted: August 19, 2012 Revision accepted: October 29, 2012 